Horizons philosophiques

\title{
La philosophie américaine : étendue et malentendus
}

\section{Francine Gagnon}

Volume 2, numéro 1, automne 1991

De Buenos Aires à Québec

URI : https://id.erudit.org/iderudit/800889ar

DOI : https://doi.org/10.7202/800889ar

Aller au sommaire du numéro

\section{Éditeur(s)}

Collège Édouard-Montpetit

\section{ISSN}

1181-9227 (imprimé)

1920-2954 (numérique)

Découvrir la revue

Citer cet article

Gagnon, F. (1991). La philosophie américaine : étendue et malentendus. Horizons philosophiques, 2(1), 115-125. https://doi.org/10.7202/800889ar d'utilisation que vous pouvez consulter en ligne.

https://apropos.erudit.org/fr/usagers/politique-dutilisation/ 


\section{La philosophie américaine : étendue et malentendus}

The only sin is limitation

R.W. Emerson

Si la philosophie est avant tout une aventure, on peut dire que sa mise en forme demande une reconnaissance minimale et une justification maximale. Quand on parle de la philosophie américaine, et en l'occurrence ici du pragmatisme, on retrouve le même type de réception amusée, à cet égard typique des régions au passé colonial quasi indélébile, où les sarcasmes et les anathèmes ont précédé ce qui s'avère aujourd'hui un mouvement fortement institué, identifié et exploré, et qui n'a plus rien à envier à la tradition européenne, malgré les engouements de circonstance pour une pensée exogène. Notre point de vue consistera à associer à toute idée un champ social d'où sont réarticulés des mythes puissants, dont celui, récurrent, de la création de l'Amérique elle-même.

En effet, au départ, pour les Américains, il y a une rupture avec la mère-patrie qu'il s'agira de panser, une émigration hors de l'Europe féodale et, au bout du traversier, un idéal qui a pour nom démocratie ou, à tout le moins, pour prénom une imagination libérale. Dans ce cas, la religion, la politique et la culture se trouvent liées dans 
une sorte de covenant où se trame déjà le récit eschatologique de l'homo americanus. Pour saisir les contours de cette terra incognita, un mot s'est greffé sur la topographie de la pensée américaine : le pragmatisme. Nous essaierons d'en retracer la genèse, même s'il faut avouer que le terme a été l'objet d'un discrédit et ceci, dès son origine. Charles Sanders Peirce en est l'inventeur dans un texte publié dans la Popular Science Monthly en 1878 et traduit l'année suivante dans la Revue philosophique. Cependant, il rebaptisa sa progéniture, hélas victime d'un kidnapping par William James, de pragmaticisme, de quoi s'assurer la main mise de ce rejeton qui connaîtra d'autres travestissements jusqu'à disparaître dans un anonymat quasi total. Peirce doit sa renaissance récente au concours d'un philosophe prestigieux, récipidendaire de la «MacArthur Foundation Award", Richard Rorty. Celui-ci a réussi à sortir la philosophie américaine du carcan du positivisme logique et de la philosophie analytique pour l'amener à renouer avec le courant continental européen.

Selon lui, les pragmatistes, et en tête de liste, John Dewey, avaient déjà compris, dans leur refus d'expansion doctrinale, les leçons d'une approche perspectiviste à la Nietzsche où l'idéal fondationnel s'éclipse au profit d'une conversation dès lors herméneutique. Au-delà de l'émulation actuelle pour ce qu'il faut désormais appeler le néopragmatisme, nous essaierons de situer l'itinéraire de ce mouvement, tout en prenant pour acquis que l'histoire est issue d'un processus collectif d'échanges où la diffusion des idées qui font école est grandement influencée par un espace réceptif. Et c'est dans cette réceptivité que réside tout le fardeau de l'énigme.

Revenons donc au début : «In the beginning the whole world was America ${ }^{1}$ " selon la phrase choc de Locke, mais encore fallait-il livrer la marchandise et se distinguer

1. John Locke, The second Treatise of Government, $n^{\circ} 49$, Indiapolis, C.B. Macpherson, ed., Hackett Publishing Cie, 1980, p. 29. 
de la tradition anglo-saxonne, prendre une tangente là où le rayonnement de la fière Albion risquait de réduire à néant les efforts réflexifs de cette petite colonie en marge d'un Nouveau Monde. C'est pourquoi, dans ces injonctions voire incantations visant à créer un espace intellectuel autonome, il est nécessaire de rappeler l'ascendance de Ralph Waldo Emerson. Même si on pouvait parler (avec Santayana) du dernier des puritains, il apparaît davantage aujourd'hui comme le premier des pragmatistes, celui qui a planté le grain américain et domestiqué les idées de son époque.

Emerson n'est pas un écrivain systématique mais plutôt édifiant au sens où ses écrits sont fragmentaires et imprégnés d'un idéalisme particulièrement vibrant au XIXe siècle. Chez lui, l'idéal prend les allures d'un perfectionnisme moral destiné à sortir le pays de sa minorité, autrement dit faire en sorte que le forum intellectuel à investir ne soit plus réduit à des sédiments de pensée provincialiste. Il est de surcroît le patriarche d'un mouvement anticonformiste appelé le transcendantalisme, terme nébuleux s'il en est un, mais qui, chose certaine, horrifiait les autorités orthodoxes de l'époque, d'autant plus qu'il s'inspira largement de la vague romantique allemande et d'une pléiade de penseurs extatiques tels Plotin, Swedenborg, le poète perse Saadi, sans oublier le Bhagavad Gîta. II s'est lié d'amitié avec l'extravagant germaniste écossais Carlyle qu'il a non seulement visité à plusieurs reprises mais avec qui il a entretenu une correspondance suivie (à partir de 1834). Emerson est même devenu son agent littéraire en Amérique, s'endettant pour publier ses écrits lapidaires. Carlyle lui rendit la politesse avec une édition anglaise de ses essais, accompagnée d'une préface ${ }^{2}$.

2. Sur les relations entre les deux penseurs, on peut consulter le livre de Kenneth Marc Harris, Carlyle and Emerson, The Long Devate, Havard U.P., 1978, de même que Representative Man, Ralph Emerson in his time de Joel Porte, Oxford U.P., 1979. Toutefois, la grande biographie demeure celle de Gay Wilson Allen, Waldo Emerson, Penguin Books, 1981. 
Les transcendantalistes ont vite essuyé les revers de la caricature, notamment à cause de quelques excentriques, pour ne pas dire illuminés, qui ont fait de la Nouvelle-Angleterre un laboratoire rousseauiste, créant des phalanstères comme New-Harmonies, Fruitlands, BrookFarm. Les uns pensaient faire pousser les récoltes sans engrais et en se passant de bêtes de somme, d'autres encore prétendaient que le mal était dans l'alimentation et que seuls les légumes qui s'épanouissaient à l'air libre étaient nobles. À bas les tubercules et vive le grenier transcendantal! Même si Emerson, en bon humaniste sentimental, a collaboré avec ces fabricants d'utopies, dirigeant un temps la revue The DIAL, son cadran s'est vite arrêté à la suite de l'échec de ses comparses, pour retourner à son œuvre, dans sa retraite de Concord. II publiera du reste les œuvres de son ami Thoreau et écrira des souvenirs sur Margaret Fuller. J'insiste un peu sur cet aspect biographique du personnage dans la mesure où il se fait luimême le chantre de ce genre littéraire : «ll n'y a pas d'histoire, tout n'est que biographie ${ }^{3}$, suivant en cela les traces de Carlyle pour qui les héros aiguisent les vertus mélioratives de l'homme. Emerson imitera son exemple avec ses Representative Men : Michel-Ange, Montaigne, Shakespeare, Goethe... surhommes à la Sur-Âme (over-soul) qui ont su transmettre l'idéal, révélant ainsi un penchant plutôt marqué pour l'individualisme et les génies épris du caprice, romantisme oblige.

On peut se demander alors quel est le lien qui unit cet idéaliste avec les pragmatistes, reconnus davantage pour leur solide sens commun, en plus d'un parti pris favorisant la méthode expérimentale, avant tout concrète et efficace. Notre enquête nous amène donc à questionner le contexte de cette époque pour faire apparaître l'arrièrefond économique, à savoir un capitalisme naissant, lequel

3. Waldo Emerson, "History", Essays First series, The complete Writing of R.W. Emerson, New York, W.H.M. Wise \& Co., p. 127. 
n'est pas sans encourager une doctrine expansive misant sur une confiance en soi (self-reliance) à toute épreuve. Or, Emerson propose justement de remplir ce programme en récusant le passé au profit d'un volontarisme appuyé, un culte de la personnalité, un optimisme et une invitation à expérimenter d'un bout à l'autre de la frontière. Desseins que les pragmatistes reprendront à leur compte, soulignant, de concert avec Emerson, l'aspect contingent, fluctuant et imprévisible de la recherche. On peut faire un rapprochement ici avec les opérations variables de l'économie de marché, tributaires du pouvoir à consolider coûte que coûte et d'un darwinisme qui traduisait bien les ambitions impérialistes de la jeune nation. Après tout, il faut savoir saisir sa chance! Par ailleurs, l'héritage de Waldo Emerson ne se limite pas uniquement à des jérémiades mais davantage à sa quote-part imposante dans l'élaboration d'une identité nationale à affirmer et affermir. II se sera battu pour que la critique culturelle émerge là où la corruption, le conformisme et la tradition faisaient rage. De plus, il aura décloisonné les savoirs, montrant en quoi l'instance morale parcourt tout le spectre social : de la religion à la science, il y a un conduit civil qui mène à un questionnement sur la façon de conduire sa vie. La leçon sera enregistrée par les pragmatistes. John Dewey deviendra le héraut de la pédagogie moderne, William James investiguera la religion et la psychologie, les plaçant en continuité avec le flux de l'expérience et Peirce s'intéressera à la communauté scientifique en tant que forum décisionnel dans la constitution du corpus savant.

Que retenir alors de la contribution d'Emerson dans I'histoire des idées en Amérique, sinon qu'il a su déborder ses frontières, démesure qui a été à la fois entendue et partagée par un autre philosophe perspicace : F. Nietzsche. Ce dernier, dans le Crépuscule des Idoles, rend d'ailleurs hommage au Yankee, célébrant sa "gaieté bienveillante et pleine d'esprit qui désarme le sérieux ${ }^{4}$ », en précisant que cette gaya scienza, est associé à Zoroastre 
(le fameux Zarathoustra!). Nietzsche, comme on le sait, écrira le Gai Savoir, consacrant l'expression emersonnienne et il dira apprécier une certaine «rudesse» américaine : "Le rire américain me fait du bien, ce genre de rudes marins à la Mark Twain. Plus rien d'allemand ne peut me faire rire 5 ." Le mythe américain savait déjà séduire, même s'il faut reconnaître que dans la chaîne des emprunts multiples, la sphère locale s'étend à mesure que les filières de l'universel sont replacées dans le cadre de leur propre histoire, une généalogie qui s'expose dans tout acte de compréhension (être avec). Bref, on peut constater à quel point la circulation des idées trouve (entre)preneur, là où tout un ensemble réceptif : (correspondances, publications multilatérales, traductions, etc.) amène les œuvres dans un circuit qui ne demande pas mieux que de donner sens aux affinités électives comme aux disparités.

Au-delà de la fascination et de l'exotisme qui persistent dans le regard porté sur les grands espaces, encore faut-il rappeler que l'Amérique de Jean Baudrillard ou même les louanges d'un Kenneth White devant l'homme des bois, Henry David Thoreau, s'ajoutent à une longue histoire d'emballements et d'excitations transatlantiques. Sait-on, par exemple, que dès le début du $X X^{\ominus}$ siècle, un scholar français, Régis Michaud, se chargera de faire connaître les œuvres du Sage de Concord, traduisant son autobiographie, publiant La pensée américaine, Autour d'Emerson, L'esthétique d'Emerson, L'âme américaine, et encore La vie inspirée d'Emerson.

II ne faudrait donc pas croire que l'exégèse fulgurante dont il est l'objet aujourd'hui soit tout à fait sortie des

4. F. Nietzsche, Le Crépuscule des idoles, $n^{\circ} 13$, Paris, éd. Denoël-Gonthier, 1970, p. 85. Emerson n'a-t-il pas écrit : “la poésie est le gai savoir», W. Emerson, The complete woks, vol. VII, Boston, Edward W. Emerson ed., Houghton Mifflin Co., 1903-1904, p. 37.

5. On retrouve cette citation dans la préface, signée par Kenneth White, à l'œuvre de Thoreau, Walden ou La vie dans les bois, Paris, Les Presses d'aujourd'hui, 1981, p. 9. 
limbes. À ce propos, il faut saluer le travail original de Stanley Cavell', dont les œuvres visent à montrer que les pensées d'Amérique méritent un traitement équitable dans la configuration mondiale des idées et que la littérature et la philosophie possèdent de multiples lieux communs, ne serait-ce que de se jouer des apparences, les traquer pour mieux saisir les fantômes de l'histoire qui restent immanents à un territoire à réinventer, inlassablement.

De ce point de vue, on pourrait dire que l'américanisme est inclus tout entier dans la notion d'initiative, d'entreprise et d' innovation en ce que les luttes des pionniers, pour apprivoiser la wilderness, contenaient déjà en germe le principe du pragmatisme tel qu'énoncé par Peirce : "Considérer quels sont les effets pratiques que nous pensons pouvoir être produits par l'objet de notre conception. La conception de tous ces effets est la conception complète de l'objet ${ }^{7}$." Cependant, maints malentendus viendront desservir les élans initiaux des trois promoteurs de cette doctrine : Peirce, James et Dewey, et on cantonnera cette méthode à la seule glorification de la valeur pratique d'une idée. Le problème est que William James aura tendance à appuyer ses propos de termes économiques pour le moins équivoques. Ainsi, il parle de «monnayer les vérités qui n'ont pour caractère commun que d'être, toutes, des idées qui paient (cash-value) ${ }^{8}$ ". Si une idée n'est vraie que si elle fonctionne, alors on peut imaginer un accueil des

6. Rappelons que Stanley Cavell a publié, dès 1972, un livre intitulé The senses of Walden, San Francisco, North Point Press, (éd. augmentée en 1981) avec un chapitre consacré à Emerson, "Thinking of Emerson", puis on retrouve In quest of the ordinary, Chicago, v. of Chicago P., 1988, This new yet unapprochable America, Lectures after Emerson after Wittgenstein, Albuquerque, Living Batch, P., 1989, et enfin Conditions Handsom and Unhandsome, The constitution of Emersoman Perfectionism, Chicago, U. of Chicago P., 1990.

7. Charles S. Peirce, «Hos to make our ideas clear», Philosophical Writings of Peirce, New York, Justus Buchler ed., Dover Publications, 1955, p. 31.

8. William James, Le pragmatisme, sixième leçon, Paris, éd. Flammarion, 1968, p. 154. 
plus favorables de la part des financiers dont le machiavélisme est loin de chanter le Blues du businessman. II importe, par conséquent, d'apporter des nuances à cet étiquetage dans la mesure où toute pensée s'articule forcément en fonction d'une conjoncture économique déterminée. Cela dit, les pragmatistes ont toujours résisté à toute forme d'association avec, et je cite James : "Le relâchement moral né du culte exclusif de la déesse-chienne la Réussite $^{9}$." En fait, le pragmatisme est la philosophie de la science expérimentale, ce qui suppose une mise à l'épreuve constante et publique des hypothèses préconisées. Aucune vérité absolue ne peut servir de crédo métaphysique ou de caution au matérialisme, le pragmatisme s'accommode du provisoire et du probable.

C'est d'ailleurs autour de cette idée de flux que le philosophe français Henri Bergson trouvera un écho à ses propres pensées mouvantes. Très près de James et agissant à titre de président de la "section intellectuelle et artistique" du Comité France-Amérique, il y prononcera un discours en 1913, où il déclare que l'idéal américain est de porter à son plus haut point "l'efficience des individus et celle de la nation", ajoutant que "le Français voyant surtout dans l'homme un être qui pense et l' Américain un être qui agit 10 ". Troublante révélation qui s'inscrit dans la foulée d'un pittoresque soigneusement entretenu, comme

9. Cette phrase percutante provient d'un discours que James prononça en 1903 devant la Ligue Anti-Impérialiste, dont il était le vice-président. On retrouve des extrait de son allocution dans R. B. Perry, The Thought and Character of William James, vol. II, Little Brown and Cie, Boston, 1936, p. 313.

10. Sur l'histoire du comité France-Amérique, fondé en 1909, mentionnons que Boutroux fut vice-président de la section “États-Unis». Pour plus d'informations sur les rapports proches et lointains entourant l'Amérique française, une référence s'impose : L'Amérique française devant l'opinion étrangère, 1756-1960 de Robert Hébert, précieuse anthologie qui nous renseigne sur les échanges et les enjeux politiques entourant la traversée de notre territoire. La citation provient des Écrits et Paroles de Bergson, P.U.F., 1958, p. 383. Je tiens à remercier le compagnon-chercheur R.H. pour avoir attiré mon attention sur ce filon et bien d'autres qui continuent de mobiliser le nerf de la recherche. 
les relations en chassé-croisé entre les deux nations, qu'il s'agisse aujourd'hui de Derrida, Lyotard contre Rorty, Cavell et hier de Boutroux, Bergson versus James et Dewey.

Plusieurs penseurs se rallieront aux positions idéalistes de James : nommons l'Anglais Schiller, les Italiens Papini et Prezzolini alors que les logiciens suivront les traces de Peirce, réputé en ce domaine : il s'agit de l'Anglais Ramsey et les Italiens Vailati et Calderoni. Néanmoins, les détracteurs n'allaient pas tarder à prononcer un verdict tranchant. Non seulement Arthur Lovejoy dénotera$t$-il treize sens différents du mot pragmatisme, remettant en cause l'existence même d'un corps de doctrines cohérent mais un concert d'invectives se succéderont, à commencer par Heidegger, puis Russell, Horkheimer, Adorno qui diront en substance, à l'instar d'Alain :

Ce nom barbare de Pragmatisme désigne seulement l'esprit technicien, qui prend pour régime de ne penser que son action et de ne recevoir pour preuve que les résultats ${ }^{11}$.

C'est pour cette raison qu'il faut applaudir à l'initiative de Richard Rorty ${ }^{12}$ qui redonnera au pragmatisme ses

11. Alain, «Pragmatisme», Études, Paris, Gallimard, 1968, p. 129. Hermas Bastien a également souscrit aux mêmes réserves : "La philosophie, toute saturée de préoccupations matérielles, les y a entraînés de son poids, et réaction naturelle, la matière, essentiellement diviseuse, a alourdi leur spéculation", "L'américanisation par la philosophiem, Notre américanisme, enquête de la Revue Dominicaine, Éd. de l'œuvre de presse dominicaine, Montréal, 1937, p. 48-49. Aujourd'hui, la perspective a radicalement changé. A témoin, le livre récent de Laurent-Michel Vacher, L'empire du moderne, Actualité de la philosophie américaine, Les Herbes Rouges, 1990, où, grâce à une bibliographie fournie et un travail d'analyse percutant, on a l'impression de vraiment découvrir l'impact énorme de la pensée américaine. Ajoutons que Sylvie Chaput a traduit l'essai d'Emerson, The American Scholar dans La petite revue de philosophie, Collège Édouard-Montpetit, vol. 6, $n^{\circ}$ 2, printemps 1985.

12. Rorty vient de voir son livre: Philosophy and the mirror of Nature traduit au Seuil (L'Homme spéculaire). En plus de Consequences of Pragmatism (U. of Minnesota P., 1984) de Rorty, il faut souligner la riche réflexion de Cornel West dont La pensée américaine contemporaine vient tout juste de paraître aux Presses Universitaires de France. 
lettres de noblesse. D'une part, il souligne la modernité des thèses antiessentialistes, lesquelles rendent caduque la théorie de la correspondance véritative entre mots et choses. Dans ce cas, la science ne détiendrait plus le monopole de la vérité, elle serait une conversation possible parmi d'autres voix, toutes aussi valables. D'autre part, en plus de sonner le glas du dogme positiviste, Rorty ravive les positions éthiques de ce qu'on peut dénommer le pragmatisme démocratique. Avec John Dewey comme porteétendard, Rorty s'arrogera le rôle de patriote moral de l'Amérique, se faisant l'apologue de la grande tradition libérale. Celle-ci possède, en effet, une histoire fortement enracinée, laquelle est condensée dans la séduisante thèse de Louis Hartz, The liberal tradition of America (1955) où l'Amérique est dès le départ située dans le cadre victorieux d'une révolution démocratique. Déjà Tocqueville notait l'absence de conditions féodales et de ce qui s'ensuit, à savoir qu'il n'y a aucun Ancien régime à combattre; en revanche cela explique également le fait que la critique sociale ait si peu d'envergure aux États-Unis. Néanmoins, le soi-disant consensus libéral s'est effrité plus d'une fois, on n'a qu'à rappeler l'esclavagisme, la lutte des femmes, les droits des minorités, la chasse aux sorcières dans les années cinquante, l'impérialisme technocrate, etc., pour constater que l'ethnocentrisme n'est pas nécessairement une réponse aux dilemmes sociaux. Pourtant, Rorty proclame tout haut : "We, Bourgeois Liberals", comme s'il s'autorisait d'un passé glorieux, autre façon d'oblitérer les stigmates laissés par la guerre du Vietnam.

Dans son dernier livre, il pousse même l'auto-célébration à son apex, en formulant le vœu que les pays du Tiers-Monde, nommément le Brésil, proposent des solutions novatrices aux Alexandriens, sous-entendre les "Ricains", bien assis sur leur confortable tranche d'Amérique. Paternalisme fort louable en effet, mais qui contient tout le désagréable de la petite tape à l'épaule, chiquenaude que nous avons fort bien connue ici, lors de ren- 
contres colloquiales avec les émissaires étrangers, plus soucieux de flatteries que d 'établir un dialogue serré et fécond.

Je conclus en souhaitant que la philosophie américaine reste tout de même un modèle dans la mesure où elle a su résister au chant des sirènes européennes. En outre, on a pu constater à quel point les enjeux territoriaux concernent et préoccupent l'Amérique et ce, depuis le premier contact avec le Nouveau Monde. Du reste, le cordon ombilical ne fut pas désentortillé aisément et il a fallu un Emerson, mais aussi un Mencken, dont le livre séminal The American Language, a contribué à suspendre l'imitation servile à la mère-patrie, repoussant ainsi un univers impérial empesé, société corsetée et surcodifiée au profit d'une langue capable désormais d'appliquer son propre vernis culturel.

De toute façon, comme l'Amérique, nos options restent ouvertes. Mais nous savons maintenant que l'écueuil le plus ardu serait d'échouer sur les rives béantes d'un exotisme charmant...bien que toujours désarmant!

FRANCINE GAGNON Collège Jean-de-Brébeuf 